\title{
Monoculturalidad en las prácticas pedagógicas en la formación inicial docente en La Araucanía, Chile'
}

\author{
Katerin Arias-Ortega ${ }^{2}$ \\ Segundo Quintriqueo M. ${ }^{2}$ \\ Vanessa Valdebenito Z. ${ }^{2}$
}

\section{Resumen}

El tema central del artículo se relaciona con las prácticas pedagógicas monoculturales en la formación inicial docente y el sistema educativo escolar. El problema que se aborda es la descontextualización curricular en la formación inicial docente. El objetivo es analizar la monoculturalidad de prácticas arraigadas a métodos y contenidos eurocéntricos occidentales, producto del legado colonial y poscolonial en Chile. La metodología consistió en una revisión de la literatura normativa y científica a nivel nacional e internacional, para lograr una comprensión y explicación del problema de la monoculturalidad en las prácticas pedagógicas. Los principales resultados aportan un estado del arte sobre el desarrollo histórico de la educación en contexto indígena e intercultural, donde se evidencia el desarrollo de prácticas pedagógicas monoculturales de manera transversal en los niveles del sistema educativo chileno. El ensayo propone algunas pistas de acción para transformar la formación inicial de profesores, a través del enfoque educativo intercultural y competencias comunicativas interculturales, con el fin de superar la monoculturalidad, el racismo hacia las personas indígenas, tanto en el medio escolar como social. Se concluye que: 1) la monoculturalidad persiste como una práctica en la formación inicial docente, lo que trae como consecuencia el desconocimiento del contexto social y cultural de los estudiantes, tanto en los formadores de profesores como en los futuros profesores; y 2) la educación escolar continúa con la transmisión de prejuicios y la construcción de actitudes racistas, perpetuando el racismo institucionalizado en contextos indígenas e interculturales.

\section{Palabras clave:}

Prácticas pedagógicas - Monoculturalidad - Formación inicial docente - Enfoque educativo intercultural.

\footnotetext{
1- Agradecimientos al proyecto FONDECYT N¹140490, Conocimientos geográficos y territoriales mapuches: una base para la formulación de contenidos educativos interculturales pertinentes y contextualizados y Al proyecto FONDEF No ID16l10350, Modelo de intervención educativa intercultural en contexto indígena, financiado por el Fondo de Fomento al Desarrollo Científico y Tecnológico.

2- Universidad Católica de Temuco (UCT), Temuco, La Araucanía, Chile. Contactos: ariaskaterin@gmail.com; squintri@uct.cl; vvaldebenito@uct.cl
}

DOl: http://dx.doi.org/10.1590/S1678-4634201711164545 


\section{Monoculturalism in pedagogical practices along basic teacher education in La Araucanía, Chile}

\section{Abstract}

The main topic of this article is related to monocultural pedagogical practices taking place in basic teacher training and the school education system. The problem covered is the curricular decontextualization in such basic teacher education. The aim is to analyze monoculturalism in practices that are deep-rooted in Western, Eurocentric methods and contents, a product of the colonial and post-colonial legacy in Chile. The methodology consisted of reviewing standard-setting and scientific literature on national and international level to better understand and explain the problem of monoculturalism in teacher education. The main results contribute to the current state of the art on historical development of education in indigenous and intercultural contexts, where the development of monocultural pedagogical practices at all levels of the Chilean educational system has become evident. This work provides some guidelines to transform basic teacher training through an intercultural education focus and intercultural education competences in order to overcome monoculturalism and the racism against indigenous people, in education but also in society at large. Conclusions are that 1) monoculturalism persists as commonplace in basic teacher training, which results in the lack of knowledge of the students' social and cultural by both the teacher educators and by the future teachers; and 2) school education continues to convey prejudices and yielding racist attitudes, perpetuating the overall institutionalized racism in indigenous and intercultural contexts.

\section{Keywords}

Pedagogical practices - monoculturalism - basic teacher training - intercultural education focus

\section{Introducción}

El problema que aquí se aborda tiene relación con las prácticas educativas monoculturales en el sistema educativo chileno en Enseñanza Parvularia, Educación Básica, Media y Superior, enmarcado desde sus inicios como un mecanismo de homogeneización de los sujetos que asisten a la institución escolar. Desde una perspectiva histórica, la escuela instaurada en la época colonial concibe la educación desde una lógica de pensamiento monocultural eurocéntrico propio de los conquistadores españoles, que construye en las personas una racionalidad, una forma de pensar y concebir el mundo (QUINTRIQUEO et al., 2014). Con ello, el conocimiento occidental es considerado como el único válido para la formación de los sujetos en el marco de una sociedad centrada en 
el orden social, moral y en los contenidos disciplinarios deseables de transmitir por los Estados nacionales (WALSH, 2000; TUBINO, 2005a; FUENTES, 2010; CORNEJO, 2012). Desde una perspectiva actual, dicha lógica de pensamiento subyace en el sistema escolar chileno como un legado de la época colonial, el cual es transmitido a los estudiantes mediante el currículum escolar, constituyéndose este, en un dispositivo que perpetúa la monoculturalidad. Por consiguiente, el currículum escolar en La Araucanía no logra enfocar la educación desde una perspectiva que dé cuenta de la diversidad social y cultural del contexto de los estudiantes.

En consecuencia, el problema de la monoculturalidad se ve reflejado en el sistema educativo chileno de manera transversal, privilegiando formas de enseñanza que dan cuenta de una hegemonía del conocimiento occidental, por sobre el conocimiento indígena, popular o campesino (TUBINO, 2011; QUILAQUEO; TORRES, 2012; QUILAQUEO et al., 2014). En el presente ensayo, sostenemos que la monoculturalidad trae como consecuencia la formación de sujetos que conciben una sola forma de comprender y dar significados a la realidad y a los hechos que ocurren en la cotidianeidad, con lo cual se reproduce el conocimiento occidental eurocéntrico en la formación de profesores. Desde esta perspectiva, queda en evidencia que en el sistema educativo en general aún no se logra romper con la lógica estructuralista, instrumental y técnica que jerarquiza el sistema escolar chileno, en el cual la monoculturalidad es su pilar fundamental.

En este contexto, en algunas universidades del Consejo de Rectores de las Universidades Chilenas (CRUCH), las carreras de Pedagogía en Educación General Básica se caracterizan por implementar el Modelo de Formación Inicial Docente basado en un enfoque por competencias. Este modelo educativo se enmarca en las reformas educativas del contexto europeo, el cual se importa a Chile y se presenta como una solución al empobrecimiento de las prácticas pedagógicas en las aulas, que carecen de modelos didácticos pertinentes al contexto sociocultural con los cuales se viene trabajando (MORENO, 2010). No obstante, el enfoque por competencia no se constituye en una solución al problema, puesto que sólo se enfoca en la dimensión académica, dando énfasis en el conocimiento disciplinario y pedagógico que se enseña. De esta manera, el enfoque por competencias se constituye en un atributo que se le otorga a los individuos en el ámbito académico y social, como un elemento que permite interacciones del sujeto con su medio laboral, material y humano (RUIZ, 2008).

Desde la perspectiva anterior, se espera que en la medida que el individuo desarrolle las competencias propuestas en su itinerario formativo, logrará cumplir con las expectativas del Estado, el cual espera formar mano de obra calificada para desenvolverse y aportar al modelo de desarrollo de la sociedad neoliberal (FERRADA; VILLENA; TURRA, 2015). Desde este punto de vista, se releva que el modelo educativo basado en un enfoque por competencias no considera las condiciones sociales y culturales en las que el docente desempeña su quehacer profesional. En este sentido, en la configuración de los itinerarios formativos en la formación inicial docente, las prácticas pedagógicas progresivas conforman un eje vertebrador de formación, donde bajo el enfoque por competencias no necesariamente se consideran los conocimientos de la realidad sociocultural en la cual se desenvolverán los futuros profesionales. 
Dentro de este marco, algunos itinerarios formativos de Pedagogía en Educación General Básica han instalado una cultura evaluativa para la mejora continua del perfil de egreso, de manera que oriente el Plan de estudios y logre consistencia entre el perfil, currículum y estrategias pedagógicas en el contexto de su Modelo Educativo Institucional (SEPÚLVEDA; MELLAD0, 2014). De este modo, los estudiantes finalizan su período formativo con un título que les habilita para trabajar en el nivel de Educación Básica y a focalizarse en una disciplina del conocimiento escolar. No obstante, no aparece reflejado en el itinerario formativo ni en el perfil de egreso, aspectos específicos asociados al reconocimiento y valoración de la diversidad social y cultural de la región de La Araucanía (TURRA; VALDEBENITO; TORRES, 2015). Además, se evidencia la falta de incorporación de saberes y conocimientos locales, campesinos o de pueblos indígenas en los itinerarios formativos (FERRADA; VILLENA; TURRA, 2015). Estos aspectos reflejan la monoculturalidad presente en la formación de los futuros docentes.

Metodológicamente, la delimitación del corpus de artículos científicos y su posterior análisis para construir este ensayo teórico, se elaboró de acuerdo al siguiente procedimiento: en un primer momento, se efectuó una revisión de artículos científicos en bases de datos en línea (Scopus, Scielo, ERIC) de revistas nacionales e internacionales. Los motores de búsqueda empleados se focalizaron con el uso de palabras clave: a) prácticas pedagógicas en contextos indígenas; b) educación escolar en contexto indígena; c) monoculturalidad y colonialidad en la educación; d) enfoque educativo intercultural; y e) competencia comunicativa intercultural en educación. En un segundo momento, se obtuvieron 100 artículos, para su revisión, lo cual permitió seleccionar 36 artículos, los que conformaron el corpus de análisis central para dar sustento a los enfoques educativos que históricamente se aplican en contextos indígenas e interculturales. Los criterios de inclusión son: 1) artículos de divulgación de resultados de investigación sobre prácticas pedagógicas en contexto indígena, publicados en Europa, Latinoamérica y Chile, en idioma inglés, francés, portugués y español; 2) artículos científicos publicados entre los años 2000-2016; también se consideraron veinticuatro libros de carácter académico y/o documentación gubernamental que tratan sobre el contenido central del ensayo; excepcionalmente se consideró un texto fundamental del año 1939, que sintetiza la historia de la educación en Chile; y 3) se incluyeron revisiones de planes de estudios de universidades que pertenecen al CRUCH y un informe de acreditación de la carrera de Pedagogía en Educación General Básica de la Universidad Católica de Temuco. Esto se complementó con el análisis de itinerarios formativos de carreras de Pedagogía de universidades chilenas, mediante la revisión de sus plataformas digitales. También se incluyó el análisis de un libro de texto escolar complementario, distribuido en el sistema educativo escolar, para contextualizar la educación escolar en La Araucanía.

El procedimiento de análisis utilizado es de contenido (KRIPPENDORF, 1990; QUIVY; CAMPENHOUDT, 1998), el cual busca develar núcleos de temas de orden abstracto, dotados de sentido, desde la perspectiva de los productores del discurso, a partir de contenidos concretos expresados en el texto y contenidos latentes (PEREZ, 2000; BISQUERRA, 2004). El análisis de la documentación utilizó una codificación abierta, identificando en una palabra 
o frase su sentido epistémico en relación al objeto de estudio, teniendo en cuenta la lógica del discurso y el contexto, para la interpretación de los contenidos centrales (QUILAQUEO; QUINTRIQUE0, 2016). Este proceso fue llevado a cabo mediante un enfoque teórico latinoamericano crítico, para comprender la evolución de la formación inicial docente, la educación escolar y las prácticas pedagógicas en contextos indígenas e interculturales.

En el presente artículo sostenemos como tesis la invisibilización de los conocimientos educativos propios de los pueblos indígenas en los itinerarios formativos de los programas de formación inicial docente. Esto podría incidir en la monoculturalidad de las prácticas pedagógicas de los futuros profesores, producto del desconocimiento del contexto sociocultural de los estudiantes.

\section{Prácticas pedagógicas en la formación inicial docente}

Las prácticas pedagógicas en la formación inicial docente son concebidas como la configuración del quehacer pedagógico que el formador de formadores lleva a cabo en la Educación Superior. El formador de formadores es concebido como parte de un colectivo, el cual media la construcción de conocimientos con otros actores sociales, para en conjunto, aprender y comprender conceptos y prácticas propias del ejercicio profesional (PALACIOS, 2000; REYES-JEDLICKI et al., 2014). Asimismo, son considerados como los especialistas que planean, implementan actividades, proyectos y programas de una determinada política educacional o quienes participan de líneas de investigación educacional que, además, incorporan la formación de tesistas de pregrado y postgrado.

En consecuencia, el formador de formadores es un agente que desarrolla los contenidos planteados en los itinerarios formativos y es el encargado de socializar un saber, contribuyendo de esta forma a implementar proyectos educativos desde las diversas áreas del conocimiento, con el propósito de generar aprendizajes en sus estudiantes, los cuales serán futuros profesores (MIRANDA; RIVERA, 2009). De esta manera, mediante las prácticas pedagógicas el formador de formadores lleva a cabo su quehacer educativo, proceso en el cual buscarían patrones que permitan teorizar y mejorar las políticas educativas en torno a la tarea de formar en los conocimientos disciplinarios y pedagógicos (JORAM, 2007; MIRANDA; RIVERA, 2009).

Desde un contexto histórico, las prácticas pedagógicas llevadas a cabo por el formador se han enmarcado en un tipo de docencia que se caracterizaba por basarse en textos consagrados, en la cual el catedrático leía, explicaba o comentaba un tema en específico (LABARCA, 1939). Entendiéndose que los sujetos que se formaban en la Universidad, ya sean filósofos, teólogos o juristas y preceptores, debían asistir a clases con los materiales necesarios para escribir lo que los catedráticos dictasen en sus facultades. De esta manera, se apoyaban en cuadernos con métodos que utilizaban como una guía para poder estudiar los contenidos posteriormente. Según la autora, además de las clases regulares, se verificaban conferencias en las cuales un maestro o un estudiante sostenían una tesis, la cual se trataba de impugnar por otros asistentes. Es así como para egresar se les solicitaba que rindiesen un examen, para el cual se les daba 24 horas de preparación. En dicho examen, se sometían a preguntas asociadas a las temáticas que un niño elegía 
con un punzón, proceso que se llevaba a cabo durante un día completo (LABARCA, 1939). Posteriormente, aprobado el examen eran habilitados para investirse. Este examen se pagaba mediante una propina. Desde el punto de vista anterior, se evidencia la supremacía teórica y metodológica en función de varias disciplinas. Primando de esta manera la memorización, proceso que trajo como consecuencia que los profesores no dominasen los aspectos prácticos asociados a conocer la realidad y las características de sus estudiantes.

Dentro de este contexto, por una parte, surge la problemática de no manejar la pedagogía como disciplina, asociado a lograr un conocimiento sobre el desarrollo físico, emocional e intelectual de los estudiantes. Por otra parte, se evidencia que la formación inicial docente se basaba en "modelos educativos, traídos del extranjero, de países como Francia y Alemania, en los cuales las disciplinas, métodos, programas y aún la enseñanza de algunos ramos, eran simples remedos y copias de los usos germánicos" (LABARCA, 1939, p. 50). Es así como la formación docente hereda un carácter centralista y uniforme, descontextualizado de la realidad social y cultural, donde se excluyen los saberes de pueblos indígenas, siendo invisibilizados en el sistema educativo. Lo anterior puede jugar un papel en la renovación descontextualizada de la formación de profesores en América Latina, como un fenómeno transversal y permanente en todos sus niveles educativos.

En Chile, dicho fenómeno se ha llevado a cabo históricamente, desde la colonia y posteriormente con el devenir del Estado, en cuyo proceso se reproduce el colonialismo donde un ejemplo de ello, es que varias universidades del CRUCH han organizado el currículo desde un modelo basado en competencias, el cual responde a estándares internacionales como es el Acuerdo de Bolonia de 1999 (FERRADA; VILLENA; TURRA, 2015). Asimismo, la política asumida por todas las instituciones formadoras de profesores del país, en alianza con el Estado, ha restringido la discusión en torno a un modelo impuesto (FERRADA; VILLENA; TURRA, 2015).

En esa perspectiva y considerando el contexto actual de renovación curricular de la formación profesional de docentes, se identifican algunos problemas: 1) omisión de otras corrientes curriculares; 2) nula descripción de supuestos teóricos que están a la base; y 3) omisión de la participación del profesorado y miembros del sistema educativo local y nacional. Este último aspecto también está asociado a la omisión de la urbanidad marginal, la diversidad cultural de los pueblos originarios e inmigrantes, entre otros (FERRADA; VILLENA; TURRA, 2015). De acuerdo a lo anterior, se observa que la formación inicial docente, tal cual se realiza en la época colonial y poscolonial, tiende a la imitación de modelos que no siempre son coherentes y consistentes con el contexto; realidad histórica, social y cultural de los estudiantes.

De esta manera, queda en evidencia que la formación en Educación Superior ha traído enraizada la copia de modelos extranjeros, fundada en la admiración a lo europeo y el menosprecio de los conocimientos de los pueblos indígenas (LABARCA, 1939; SARANGAPANI, 2014). Asimismo, se refleja que los modelos educativos extranjeros responden a contextos sociales y culturales diferentes al contexto nacional y regional. Los resultados que se han obtenido de este proceso son la instalación de prácticas pedagógicas monoculturales, las cuales no estimulan el pensamiento 
crítico y reflexivo de los futuros profesores (QUINTRIQUEO et al., 2016). Esto ocurre porque en los itinerarios formativos no se reconoce la diversidad social y cultural que caracteriza a la sociedad chilena. Es así como desde una revisión de los planes de estudios se observa una falta de objetivos claros en la formación inicial docente, si se tiene como principio de base que los modelos educativos deben responder a las necesidades propias de las sociedades, pueblos y culturas, donde se llevan a cabo dichos procesos educativos.

De acuerdo a lo anterior y desde el aspecto de gestión curricular, en la formación inicial docente se observan patrones históricos repetitivos. Por ejemplo: 1) los estudiantes deben desarrollar un itinerario formativo, el cual está fundado en un modelo por competencias traído desde el extranjero; 2) las prácticas pedagógicas que lleva a cabo el formador de formadores se fundan en una cátedra oral, en la cual los futuros profesores deben tomar apuntes respecto de los conocimientos teóricos que se enseñan; y 3) la formación profesional y académica está basada en competencias genéricas y específicas asociadas al saber, al saber hacer y al saber ser y convivir. Dichas competencias son verificadas y validadas en distintos momentos de la formación mediante la evaluación de los productos (informes, presentaciones, indagaciones, pruebas, entre otras). Como se observa, las prácticas pedagógicas en el marco de la formación inicial continúan siendo de carácter monocultural.

\section{Prácticas pedagógicas monoculturales}

Las prácticas pedagógicas monoculturales en el sistema educativo chileno, en los diferentes niveles de educación, han traído como consecuencias acciones educativas descontextualizadas a los saberes y conocimientos propios de los estudiantes. En esa perspectiva, aun cuando en la actualidad se trata de contextualizar las prácticas pedagógicas y los recursos didácticos, de igual forma se transmiten prejuicios y actitudes racistas. Tal es el caso de la lectura literaria La princesa murta (SCHKOLNIK, 2012), que es entregada a los estudiantes de primero y segundo año de Educación Básica en La Araucanía. La finalidad de la lectura es dar a conocer las características de la murta (fruto silvestre nativo de la región), para contextualizar algunos saberes y conocimientos propios de los estudiantes. Al analizar el contenido del texto constatamos que al aparecer personajes mapuches se les asocia con adjetivos calificativos como: "el más gordo de los mapuchitos [...]", "gritaba una indiecita [...]”, "los pequeños mapuches corrían muy rápido [...]", "mapuchito glotón [...]" "muy pronto los indiecitos se olvidaron de la huemul" (SCHKOLNIK, 2012, p. 16-20). Es así como queda en evidencia la persistencia de la transmisión de prejuicios y actitudes racistas hacia los pueblos indígenas en la educación escolar, construyendo representaciones sociales negativas hacia la población mapuche, perpetuando el racismo institucional y las prácticas pedagógicas monoculturales.

Este problema, por una parte, se relaciona con la formación de docentes desde la lógica del conocimiento occidental, perpetuando el círculo del monoculturalismo en los recursos de enseñanza. Por otra parte, esto podría deberse a que en la formación de los futuros profesores no se les prepara en la construcción de recursos didácticos propios que 
respondan al contexto sociocultural de sus estudiantes. De esta forma, al no poseer estas competencias, tenderían a reproducir sin cuestionamientos y mayor reflexión lo que plantean los planes, programas y textos complementarios, los cuales explícita o implícitamente reproducen ideologías prejuiciadas y racistas, fundadas en el colonialismo que está a la base en el sistema educativo. Desde esta perspectiva, algunas investigaciones indican que los profesores creen que no han sido preparados para enseñar a niños de zonas cultural y lingüísticamente diferentes (BANKS, 2015; FERRADA; VILLENA; TURRA, 2015). En este contexto, y en base a la revisión de la literatura, se podría inferir que el desconocimiento de los docentes en ejercicio sobre la realidad sociocultural de sus estudiantes se debe a creencias y prejuicios construidos desde la educación escolar (CAUSEY; THOMAS; ARMENTO, 2000; RAY; BOWMAN, 2003; MUJAWAMARIYA; MOLDEVEANU, 2006; TURRA; VALDEBENITO; TORRES, 2015). De ahí que lo más probable es que las prácticas pedagógicas del futuro profesor serán descontextualizadas, perpetuando la negación de los saberes y conocimientos propios en la educación escolar. Teniendo como base que los futuros profesores no han sido formados en saberes y conocimientos diferentes al conocimiento occidental, institucionalizado en el sistema educativo escolar.

En esa perspectiva, los profesores que no han recibido una formación para desempeñarse en contextos indígenas e interculturales se convierten y/o se subsumen en la escuela monocultural (SARANGAPANI, 2014). Dichos profesores se han caracterizado por ser autoritarios, que fundan los procesos de enseñanza-aprendizaje desde una educación escolar monocultural que sólo busca que los niños memoricen los contenidos, mediante la repetición (WANSINK et al., 2015). La finalidad es que los estudiantes se transformen en seres obedientes, que realicen las actividades que se les encargan de manera sistemática y ordenada. Por ejemplo, en el caso de la India, se han vivido procesos muy similares a los que se han dado en Chile, en que las prácticas pedagógicas buscan formar sujetos bajo una sola forma de pensar y actuar (SARANGAPANI, 2014). Ello refleja la necesidad de los Estados-nación por continuar perpetuando el conocimiento occidental eurocéntrico, mediante prácticas pedagógicas monoculturales.

En el marco del currículum real, se observa que las evaluaciones como la disertación, pruebas escritas y talleres, se aplican en función del producto y como práctica de homogeneización del aprendizaje de los estudiantes (MEIREU, 2001). Por ejemplo en el contexto chileno, las pruebas del Sistema de Medición de Calidad de la Enseñanza [SIMCE], Prueba de Selección Universitaria [PSU], y la Prueba Inicia (que rinden los profesores al egresar de una carrera de educación) no consideran el contexto al cual pertenecen los sujetos que participan de la evaluación. Esta práctica introduce en forma progresiva en la mentalidad objetiva y subjetiva de los sujetos, una idea de producción y progreso que no siempre es coherente y consistente, con el marco social y cultural de las familias y comunidades mapuches.

De acuerdo a lo anterior, queda en evidencia que las prácticas pedagógicas monoculturales se continúan perpetuando en el sistema educativo chileno, dado que no se ha logrado romper con el legado colonial asociado a una educación monocultural. 


\section{Educación monocultural}

La educación monocultural es un pilar de la sociedad dominante, la cual se funda en el conocimiento occidental, históricamente transmitido como hegemonía hacia los conocimientos educativos propios de las familias mapuches (WALSH, 2000; TUBINO, 2005a; CORNEJO, 2012; QUINTRIQUEO et al., 2016). Se sostiene que el actual sistema educativo, en cualquiera de sus niveles, es un legado de la época colonial, el cual está asociado a la homogeneización y centralismo que se le ha otorgado a la educación, desde sus orígenes hasta la actualidad.

Este tipo de educación es entendida como aquella que busca la homogeneización del individuo, mediante la transmisión del conocimiento de corte eurocéntrico occidental, considerado como único, válido y científico, y que por tanto, se constituye en un statu quo existente en la sociedad actual. Según Sarangapani (2014) la enseñanza tradicional que se entrega en las escuelas coloniales y poscoloniales, sólo considera los conocimientos occidentales y el idioma oficial de los conquistadores (inglés, español, portugués, francés), como contenidos que los sujetos deseablemente deben aprender. De esta forma, se excluye del sistema educativo todo aquel conocimiento que no sea considerado válido, en este caso el conocimiento de los pueblos indígenas, por lo cual no se incorpora a la enseñanza escolar. En este sentido, en la enseñanza escolar prima el conocimiento memorístico, instrumental y técnico, el cual es controlado mediante la producción y evaluación cuantificable, respecto de lo que supuestamente saben los sujetos que están en el sistema escolar actual (QUINTRIQUEO et al., 2016a).

En consecuencia, la educación monocultural que se encuentra arraigada en el sistema educativo chileno es el reflejo de las diversas sociedades donde el currículum escolar se constituye en uno de los factores más significativos que afectan los procesos de enseñanzaaprendizaje en la escuela (ATTA-ALLA, 2012). Entendiendo al currículum escolar en su dimensión formal, real y oculta: 1) el currículum formal está constituido por las leyes que asignan los fines de la educación pública, los programas de estudio, los métodos en los cuales se debe desarrollar y que pretenden asistir o regir la acción pedagógica; 2) el currículum real corresponde al proceso que se lleva a cabo durante el desarrollo de la actividad en aula, asociado a lo que realmente se desarrolla en las actividades educativas escolares en una relación educativa que se establece entre el profesor-estudiantes y los contenidos educativos deseables de aprender y enseñar; y 3) el currículum oculto está referido a los aprendizajes que no aparecen programados por la institución escolar, por el currículum escolar, al menos no explícitamente, pero que son transmitidos en el aula, donde se constituyen en un conjunto de saberes para desenvolverse adecuadamente en la sociedad (PERRENOUD, 1994).

En concordancia con lo anterior y al revisar las bases curriculares del Ministerio de Educación (CHILE, 2012) como los planes de estudios de la formación de profesores de Educación General Básica de algunas universidades del CRUCH, se observa que los contenidos educativos definidos en el currículum propuesto por Perrenoud (1994) continúan siendo de carácter monocultural en cada una de sus dimensiones. Asimismo, se evidencia en el marco del currículum escolar del sistema educativo chileno, que la educación aún 
está arraigada a sus finalidades históricas, desde su instauración en contextos indígenas (LABARCA, 1939; SERRANO; PONCE DE LEÓN; RENGIFO, 2012). Estas finalidades son: 1) la civilización de la población indígena, la cual trae como consecuencia un desarraigo cultural sistemático y progresivo de las poblaciones indígenas, en desmedro de saberes educativos propios en las familias y comunidades; 2) la evangelización como una primera forma de escolarizar a las nuevas generaciones de mapuches, la cual suprime los valores vitales de la identidad sociocultural como es su lengua y prácticas socio-religiosas, asociadas a prácticas monolingües, en las cuales se funda el proceso de enseñanzaaprendizaje; 3) la colonización que impone a las nuevas generaciones la racionalidad occidental sobre la forma de ver y comprender el mundo, la forma de comprender las relaciones sociales y culturales; y 4) la monoculturalidad de la escuela, la que trae como consecuencia una asimetría en la relación de poder entre el conocimiento occidental y el conocimiento de los pueblos indígenas (QUINTRIQUE0, 2010).

Desde esta perspectiva, el sistema educativo se caracteriza por la negación de las minorías en el contexto escolar. De esta manera, a nivel mundial y local los vínculos entre la escolarización y la colonización traen como consecuencia la jerarquización y segregación de las etnias. Históricamente se observa la resistencia de la escuela para incorporar la pluralidad cultural de los estudiantes, proceso que no ocurre solamente en la institución escolar, sino también en la educación universitaria (BISHOP et al., 2009; SALÜN, 2013; SARANGAPANI, 2014; MAMPAEY; ZANONI, 2015).

En este sentido, en Chile como en otras partes del mundo, aún podemos evidenciar que la educación sigue arraigada al colonialismo, lo cual genera la invisibilización de todo aquel tipo de conocimiento que no provenga de la sociedad occidental (QUINTRIQUEO et al., 2016b). Asimismo, las políticas públicas han ayudado al encubrimiento del colonialismo, bajo el discurso de una educación de calidad para todos, la cual busca lograr mejores expectativas de vida en los sujetos. De esta manera, el colonialismo ha sido estratégicamente encubierto en la escuela, el cual ha tenido como resultado la invisibilización del propio colonialismo, no reconociendo la lucha de poder de los pueblos indígenas respecto del reconocimiento de sus saberes y conocimientos (SANTOS, 2009; SARANGAPANI, 2014). En esta perspectiva, la educación escolar que se lleva a cabo en Chile es en gran parte la responsable de las representaciones simbólicas, que transmiten patrones que coinciden con la cultura de los grupos dominantes de la sociedad. Aquí se observa, empírica y teóricamente, la idea de orden social, orden moral, progreso y producción económica como un telón de fondo (SALÜN, 2013), que impone la hegemonía del conocimiento occidental en la escuela y la sociedad.

De acuerdo a lo anterior es que los sujetos que han sido educados en el sistema educativo chileno son formados bajo una lógica técnica e instrumentalista, la cual no considera las diferencias sociales y culturales (FERRADA; VILLENA; TURRA, 2015). Por tanto, la escuela se transforma en una empresa desarrollada e instaurada por la sociedad colonizadora, que apoya el mantenimiento de las estructuras de subordinación cultural y social, que va más allá de la época colonial (QUINTRIQUEO et al., 2014).

Según Mampaey y Zanoni (2015) este colonialismo encubierto mediante la monoculturalidad está asociado al racismo institucional, el cual reproduce prácticas 
monoculturales en la institución escolar. Existen tres tipos de prácticas características de una educación desde un enfoque monocultural: 1) la práctica monolingüe basada en la lengua oficial del grupo social dominante en la cual se desarrollan los procesos de enseñanza-aprendizaje y que niega la incorporación de lenguas de grupos sociales minoritarios al sistema educativo escolar; 2) las prácticas que excluyen las minorías ya sean religiosas, étnicas, en el desarrollo de las clases; y 3) el uso del currículum de corte eurocéntrico occidental, el cual desarrolla los conocimientos y valores que la sociedad occidental considera válidos, invisibilizando los saberes, conocimientos y valores que poseen los estudiantes de diferentes culturas (MAMPAEY; ZANONI, 2015; QUINTRIQUEO et al., 2016b). Esto trae como consecuencias relaciones desiguales de poder entre los individuos que convergen a diario en la institución escolar (QUINTRIQUEO et al., 2015). Por lo tanto, la educación desde un enfoque monocultural, tiene como finalidad que los estudiantes del sistema educativo, ya sean niños mapuches y no mapuches, o inmigrantes, adquieran las normas de comportamiento, valores y conocimientos de la sociedad dominante, reproduciéndose de esta forma el racismo institucional.

En este contexto, se hace necesario formar a los futuros profesores en competencias interculturales, esto como una acción para contrarrestar la monoculturalidad y, a la vez, como una alternativa que permita mejorar la contextualización de la formación de los profesores. En este contexto, diferentes investigaciones a nivel internacional (NIETO; SANTOS, 1997; CARIGNAN; SANDERS; POURDAVOOD, 2005; PAYET, 2006; CANEN, 2007; AKKARI, 2009; BARTEL-RADIC, 2009; MONDOVEALU, 2009; MAMPAEY; ZANONI, 2015) y a nivel nacional (QUILAQUE0; TORRES, 2012; TURRA; FERRADA; VILLENA, 2013; QUINTRIQUEO et al., 2014) han entregado pistas de cómo se podría transformar estas prácticas pedagógicas monoculturales en la formación inicial docente. Con este propósito, se propone en la formación inicial docente formar en competencias interculturales, las cuales le permitirán al futuro profesor desarrollar prácticas pedagógicas contextualizadas. Estas competencias interculturales están asociadas a: 1) la necesidad de llevar a cabo la formación inicial docente desde un enfoque educativo intercultural, teniendo en cuenta la realidad social y cultural del país; y 2) la necesidad de que la formación inicial docente contribuya al desarrollo de habilidades específicas relacionadas a la incorporación de las características socioculturales de la población estudiantil (MOLDOVEANU, 2009; CONTRERAS et al., 2010). Es así como un enfoque educativo intercultural permite el estudio y comprensión de personas pertenecientes a diferentes sociedades y culturas, con el fin de construir un saber multipolarizado para superar la discriminación y el racismo (ESSOMBA, 2012). Este enfoque se concibe como una alternativa que favorece y permite la relación de saberes y conocimientos de los pueblos indígenas con el conocimiento escolar.

Esto permitirá que el futuro profesor desarrolle sus prácticas pedagógicas contextualizadas con la finalidad de desarrollar y conducir situaciones de aprendizajes, con sentido en los procesos de enseñanza-aprendizaje de acuerdo a las características de los estudiantes. Esto es posible mediante una reflexión orientada desde un marco de análisis como referencia para reflexionar sobre las propias prácticas. 
Esquema 1 - Marco de análisis de prácticas pedagógicas en contextos interculturales.

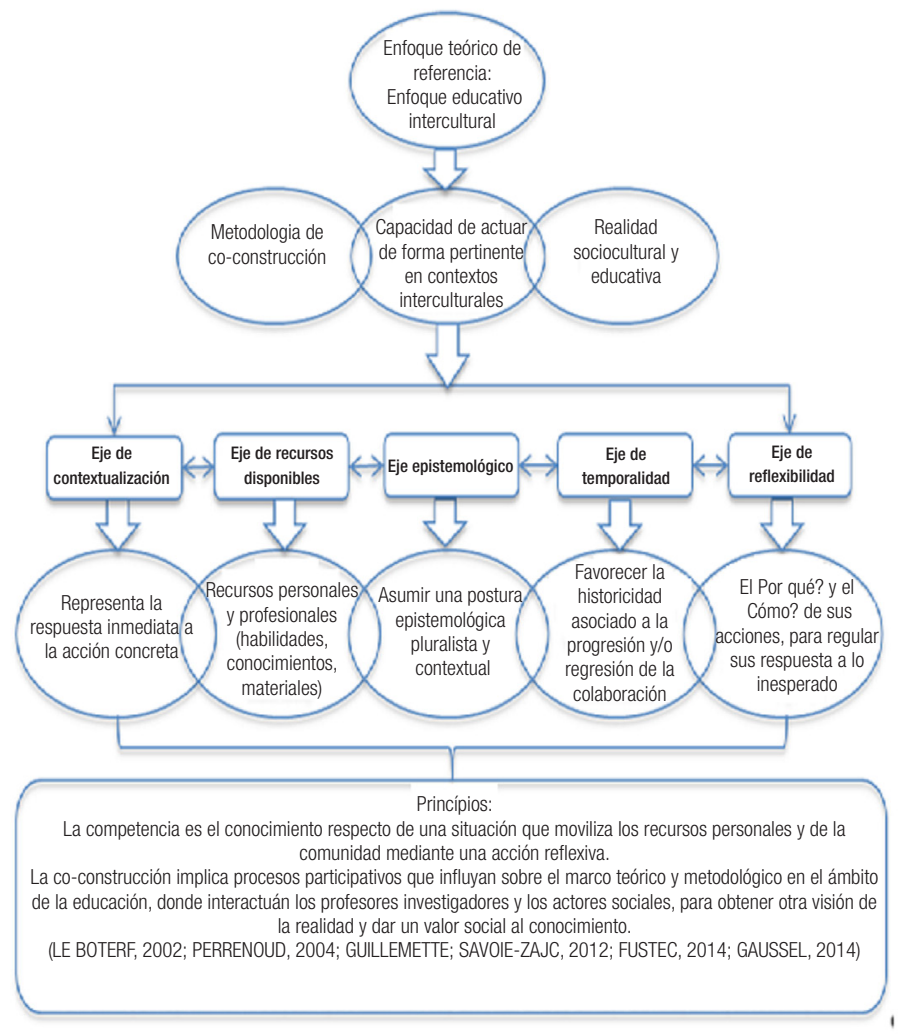

Fuente: Elaboración propia.

Según el esquema 1, el análisis de las prácticas pedagógicas requiere de un marco de referencia que explicite una base teórica desde un enfoque educativo intercultural, articulada a una metodología de co-construcción del conocimiento escolar y a la realidad sociocultural y educativa de los estudiantes. En este sentido, el futuro profesor podría desarrollar competencias profesionales interculturales, entendidas como la capacidad de actuar de manera pertinente en un contexto profesional, fundadas en cinco ejes principales: 1) el eje de la contextualización de su práctica pedagógica, referido a la capacidad de responder de manera adecuada a situaciones problemáticas en un contexto determinado; 2) el eje de los recursos, asociado a la existencia de materiales educativos, habilidades, actitudes, competencias y conocimientos sobre las políticas educativas. También desarrollar capacidades de investigación y observación para la comprensión del fenómeno educativo, tomando distancia del aspecto emocional, para comprender mejor un objetivo educativo final; 3) eje epistemológico, en donde es necesario asumir el conocimiento escolar dominante como una característica de 
la educación. Desde una epistemología pluralista y contextual, los actores de la comunidad educativa deben ser capaces de conocer e incorporar las epistemologías indígenas que se encuentran en interacción constante en la sala de clases, puesto que cada sujeto es portador de saberes y conocimientos propios. Esto implica generar procesos de interacción, negociación y ajuste constante entre los conocimientos teóricos y prácticos en la relación educativa que se establece con los estudiantes; 4) eje de temporalidad, referido a la preparación y socialización con respecto a la progresión de los estudiantes, tomando las medidas necesarias para reorientar el proceso de intervención, en el caso que sea necesario. Es decir, en la interacción profesor-estudiante interviene la naturaleza pedagógica, didáctica, psicológica, social e intersubjetividad propia de cada sujeto en la construcción de conocimientos; y 5) el eje de la reflexibilidad, referido a la capacidad del profesor para construir un marco de análisis sólido, actualizado y flexible, que le permita entender sus propias prácticas pedagógicas, desde un enfoque didáctico del conocimiento disciplinar y práctico, para comprender el progreso del aprendizaje de sus estudiantes en relación a un objeto de conocimiento, asociadas al ¿Por qué? y ¿Cómo? de sus acciones y ¿Qué transformaciones son posibles para contextualizar la educación al contexto social y cultural de los estudiantes? (LE BOTERF, 2002; LENOIR, 2009; PETIGNAT, 2015; QUINTRIQUEO et al., 2016b). Por lo tanto, en la medida que el futuro docente desarrolle competencias profesionales interculturales, podría ajustar de forma crítica sus prácticas pedagógicas al contexto social y cultural en el cual se desenvuelve, para generar la transformación de la realidad social, mediante la emancipación intelectual de sus estudiantes.

\section{Reflexiones finales}

De acuerdo a la literatura revisada, los planes de formación de algunas universidades del CRUCH y Bases Curriculares del Ministerio de Educación evidencian una invisibilización histórica de los saberes y conocimientos educativos de los pueblos indígenas, locales o campesinos en los itinerarios formativos, planes de formación y en el currículum escolar. Sostenemos que esta situación podría ser consecuencia de un racismo científico y académico que existe en las instituciones de Educación Superior, sea en forma explícita o encubierta, producto de la racionalidad colonial del saber y del poder que históricamente se ha transmitido en la educación escolar en contextos de colonización. Dicha racionalidad del conocimiento científico disciplinario es transmitido en la formación inicial docente por los formadores de formadores como un conjunto de prácticas que omiten o no consideran los saberes y conocimientos educativos propios al contexto sociocultural de los estudiantes en tres niveles: en la educación superior, en la educación escolar y en la educación familiar; en este último nivel se realiza mediante la socialización primaria progresivamente monolingüe y monocultural que han adquirido los padres en su proceso de escolarización.

Una posible solución al problema es que las universidades avancen en procesos de interculturalización de las prácticas pedagógicas que ofrecen. Para ello, es necesario 
que la educación logre vincularse con el medio social y cultural en el cual se encuentra inserta. De esta manera, se podrá avanzar en visibilizar el carácter monocultural y hegemónico del conocimiento occidental, lo cual permita plantearse los desafíos para una educación con base en un pluralismo epistemológico. Así, los distintos actores del medio educativo podrían avanzar en la construcción de relaciones educativas que consideren el aspecto cultural, social, natural y político propio, que inciden en la construcción de conocimiento de sus estudiantes. Desde esta mirada, es necesario que las prácticas pedagógicas se caractericen por: 1) centrar la gestión educativa en el aprendizaje de los estudiantes desde su marco social y cultural propio, en articulación con el conocimiento occidental; 2) ser consciente de que en el proceso de formación, los conocimientos que se adquieren y construyen, ya sean disciplinares o pedagógicos, son producto de una construcción social y, por lo tanto, son permeables a la realidad social y cultural del entorno en el cual se desenvuelven los estudiantes; 3) promover la descolonización del conocimiento occidental que está arraigado en el sistema de Educación Superior, mediante la incorporación progresiva de otras formas de construcción de conocimiento, en la formación inicial, considerando la propia episteme y métodos educativos indígenas; 4) focalizar la formación de los estudiantes en el desarrollo de capacidades para investigar, cuestionar, revalorizar y potenciar los saberes locales como contenidos educativos pertinentes para enriquecer la formación de estudiantes indígenas y no indígenas; y 5) orientar la formación profesional desde un enfoque educativo intercultural para desarrollar una postura crítica, reflexiva y autónoma, respecto de su formación y responsabilidad con su contexto local, regional y nacional. Para ello, es necesario que se consideren los significados que se construyen en la interacción social entre formador de formadores, futuros profesores, escuela, familia y comunidad, puesto que son estos actores sociales quienes están implicados en los procesos educativos escolares. Sostenemos que mediante dichos desafíos podríamos avanzar en prácticas pedagógicas más contextualizadas a la realidad sociocultural de los estudiantes para contrarrestar progresivamente tanto el monoculturalismo como el racismo científico arraigado en las instituciones que imparten educación en contextos indigenas e interculturales.

Desde esta perspectiva, se considera que las relaciones entre formación inicial docente y ejercicio profesional docente aparecen como uno de los principales vértices para lograr una incorporación de los conocimientos educativos indígenas a la Educación Parvularia, Básica, Media y Superior. Sostenemos que estos conocimientos educativos deberían sustentarse en resultados de investigaciones empíricas que develan su epistemología propia para contextualizar la formación de profesores y la educación escolar en contextos indígenas, mediante un enfoque educativo intercultural. Consideramos que, al mejorar la formación inicial docente, mejorarán también las prácticas pedagógicas de los profesores en la escuela, y en consecuencia, mejorará la calidad de los aprendizajes escolares en perspectiva intercultural de los estudiantes en La Araucanía. 


\section{Referencias}

AKKARI, Abdeljalil. Introduction aux approches interculturelles en éducation. Genève: Université de Genève, 2009.

ATTA-ALLA, Monir. Egypt education system: a monocultural education in a multicultural society. Journal of Sociological Research, Las Vegas, v. 3, n. 2, p. 476-488, 2012.

BANKS, Tachelle. Teacher education reform in urban educator preparation programs. Journal of Education and Learning, Toronto, v. 4, n. 1, p. 60-71, 2015.

BARTEL-RADIC, Anne. La compétence interculturelle: état de l'art et perspectives. Management International, Montrêal, v. 13, n. 4, p. 11-26, 2009.

BISHOP, Russell et al. Te kotahitanga: addressing educational disparities facing maori students in New Zealand. Teaching and Teacher Education, Amsterdam, v. 25, n. 30, p. 1-9, 2009.

BISQUERRA, Rafael. Metodología de la investigación educativa. Madrid: La Muralla, 2004.

CANEN, Ana. Multiculturalism and a research perspective in initial teacher education: posible dialogues. Policy Futures in Education, Auckland, v. 4, n. 4, p. 519-534, 2007.

CARIGNAN, Nicole; SANDERS, Michael; POURDAV00D, Roland. Racism and ethnocentrism: social representations of preservice teachers in the context of multi-and intercultural education. International Journal of Qualitative Methods, Alberta, v. 4, n. 3, p. 1-19, 2005.

CAUSEY, Virginia; THOMAS, Christine; ARMENTO, Beverly. Cultural diversity is basically a foreign term to me: the challenges of diversity for preservice teacher education. Journal Teaching and Teacher Education, Amsterdam, v. 16, p. 33-45, 2000.

CHILE. Ministerio de Educación. Estándares orientadores para carreras de pedagogía en educación media. Santiago de Chile: Mineduc, 2012.

CONTRERAS, Inés et al. La escuela como espacio para aprender a enseñar: visiones desde los programas de formación de profesores de educación media. Estudios Pedagógicos, Valdivia, v. 36, n. 1, p.85-105, 2010.

CORNEJO, Juan. Educación, interculturalidad y ciudadanía. Educar em Revista, Curitiba, n. 43, p. 239254, 2012.

ESSOMBA, Miquel. Inmigración e interculturalidad en la ciudad: principios, ámbitos y condiciones para una acción comunitaria intercultural en perspectiva europea. Barcelona: Graó, 2012.

FERRADA, Donatila; VILLENA, Alicia; TURRA, Omar. Transformar la formación, las voces del profesorado. Concepción: Universidad Católica de la Santísima Concepción, 2015. 
FUENTES, Rocío. Convergencias y divergencias en dos discursos sobre la educación intercultural. Cuicuilco, México, DF, v. 48, n. 17, p. 165-189, 2010.

FUSTEC, Klervi. Processus multi-échelles, enjeux environnementaux et construction étatique: le cas de l'autorité palestinienne, des politiques de gestion de l'eau et du changement climatique. Montpellier: Université Paul Valéry, 2014.

GAUSSEL, Marie. Les enjeux de la construction d'une histoire scolaire commune. Dossier de Veille de I'IFÉ, Lyon, n. 109, p. 1-28, 2014.

GUILLEMETTE, Suzanne; SAVOIE-ZAJC, Lorraine. La recherche-action et ses rapports de co- construction de savoirs et de formation dans une perspective de professionnalisation entre acteurs praticiens et chercheurs. Formation et Profession, Montreal, v. 3, n. 20, p. 14-25, 2012.

JORAM, Elana. Clashing epistemologies: aspiring teacher's, practicing teacher's and professor's beliefs about knowledge and research in education. Teaching y Teacher Education, Amsterdam, v. 23, p. 123135, 2007.

KRIPPENDORFF, Klaus. Metodología de análisis de contenido: teoría y práctica. Barcelona: Paidós, 1990.

LABARCA, Amanda. Historia de la enseñanza en Chile. Santiago de Chile: Universidad de Chile: Imprenta Universitaria, 1939.

LE BOTERF, Guy. Développer la compétence des professionnels. 4. ed. Paris: Éditions d’Organisation, 2002.

LENOIR, Yves. L'intervention éducative, un construit théorique pour analyser les pratiques d'enseignement. Nouveaux Cahiers de la Recherche en Éducation, Quebec, v. 12, n. 1, p. 9-29, 2009.

MAMPAEY, Jelle; ZANONI, Patricia. Reproducing monocultural education: ethnic majority staff's discursive constructions of monocultural school practices. British Journal of Sociology of Education, v. 37, n. 7, p. 928-946, Inglaterra, 2015. Disponible en: <http://dx.doi.org/10.1080/01425692.2014.1001059> . Acceso en: 12 may 2016.

MEIREU, Phillipe. La opción de educar, ética y pedagogía. España: Octaedro, 2001.

MIRANDA, Christian; RIVERA, Pablo. Formación permanente de profesores: ¿Quién es el formador de formadores? Estudios Pedagógicos, Valdivia, v. 35, n. 1, p. 155-169, 2009. Disponible en: <http://www. scielo.cl/scielo.php?script=sci_arttext\&pid=S0718-07052009000100009\&lng=es\&nrm=iso>. Acceso en: 13 jun. 2017.

MONDOVEALU, Mirela. Pour une conceptualisation de la compétence multiculturelle de l'enseignant. Education Canadienne et Internationale, v. 38, n. 2, p. 54-74, 2009.

MORENO, Tiburcio. Reseña de educar por competencias, ¿qué hay de nuevo? Revista Mexicana de Investigación Educativa, México, DF, v. 15, n. 44, p. 289-297, 2010. 
MUJAWAMARIYA, Donatille; MOLDEVEANU, Mirela. Qu'apprennent les étudiants-maîtres au sujet de la gestión de classe multiculturelle pendant leurs stages d’enseigment? In: MUJAWAMARIYA, Donatille (Org.). L’éducation multiculturelle dans la formation des enseignants au Canada: dilemmes et défis. Berne: Peter Lang, 2006. p. 153-182.

NIETO, Sonia; SANTOS, Miguel. Formación multi/intercultural del profesorado: perspectiva en los Estados Unidos y en España. Teoría de la Educación, Salamanca, v. 9, p. 55-74, 1997.

PALACIOS, María. El poder para cambiar nuestra práctica docente. In: CONGRESO INTERNACIONAL DE FORMACIÓN DE FORMADORES, 3., 2000. CONGRESO NACIONAL DE INSTITUTOS SUPERIORES, 2000, Urubamba, Cusco. Libro de ponencias... Lima: Industrial Gráfica, 2000. p. 23-33.

PAYET, Jean-Paul. L'interculturel est-il soluble dans la modernité? Fragilités et défis de la formation des enseignants à la complexité culturelle. Formation et pratiques d'enseignement en questions. Revue des HEP de la Suisse Romande et du Tessin, Ontário, n. 4, p. 205-215, 2006.

PÉREZ, Gloria. Investigación cualitativa: técnica de análisis de datos. v. 2. Madrid: La Muralla, 2000.

PERRENOUD, Phillipe. Desarrollar la práctica reflexiva en el oficio de enseñar. Barcelona: Graó, 2004.

PERRENOUD, Phillipe. La formation des enseignants entre théorie et pratique. Paris: L' Harmattan, 1994.

PETIGNAT, Pierre. L'analyse des pratiques en formation initiale d'enseignants: quelles perceptions, quelles mises en perspective? Revue de L'analyse de Pratiques Professionnelles, n. 5, p. 3-15, 2015.

QUILAQUEO, Daniel et al. Saberes educativos mapuches: aportes epistémicos para un enfoque de educación intercultural. Revista Chungará, Arica, v. 46, n. 2, p. 271- 283, 2014.

QUILAQUEO, Daniel; QUINTRINQUEO, Segundo. Métodos educativos Mapuches: retos de la doble racionalidad educativa. Aportes para un enfoque de educación intercultural. Temuco: Universidad Católica de Temuco, 2017.

QUILAQUEO, Daniel; TORRES, Héctor. Multiculturalidad e interculturalidad: desafíos epistemológicos de la escolarización desarrollada en contextos indígenas. Alpha, Osorno, n. 37, p. 285-300, 2012.

QUINTRIQUEO, Segundo. Implicancias de un modelo curricular monocultural en contexto mapuche. Santiago de Chile: Lom. 2010.

QUINTRIQUEO, Segundo et al. Competencia comunicativa intercultural: formación de profesores en el contexto poscolonial chileno. Alpha, Osorno, no prelo, 2016b.

QUINTRIQUEO, Segundo et al. Conocimiento educativo en el contexto escolar y en la educación familiar mapuche: principales tensiones epistemológicas. Revista Brasileira de Educação, Rio de Janeiro, no prelo, 2015. 
QUINTRIQUEO, Segundo et al. Formación del profesorado en educación intercultural en América Latina: el caso de Chile. Revista Electrónica Interuniversitaria de Formación del Profesorado, Murcia, v. 17, n. 2, p. 201-217, 2014.

QUINTRIQUEO, Segundo et al. Interculturalidad en la formación inicial docente: invitación a construir un dialogo intercultural. Temuco: Universidad Católica de Temuco, 2016a.

QUIVY, Raymond; CAMPENHOUDT, Luc Van. Manual de investigación en ciencias sociales. México, DC: Limusa, 1998.

RAY, Aisha; BOWMAN, Bárbara. Learning multicultural competence: developing early childhood practitioners' effectiveness in working with children from culturally diverse communities. Chicago: [s. n.], 2003. Final report to the A. L. Mailman Family Foundation. Center for Race, Class, and Culture in Early Childhood, Erikson Institute.

REYES-JEDLICKI, Leonora et al. Subjetividades y saberes docentes en el sistema educativo chileno: un análisis desde las concepciones de formadores de profesores. Estudios Pedagógógicos, Valdivia, v. 40, n. especial, p. 183-203, 2014. Disponible en: <http://www.scielo.cl/scielo.php?script=sci_ arttext\&pid=S0718-07052014000200011\&lng=es\&nrm=iso >. Acceso en: 13 jun. 2017.

RUIZ, Guido. Reflexiones y definiciones desde la teoría biológica del conocimiento: aprendizaje y competencia en la universidad actual. Estudios Pedagógicos, Valdivia, v. 34, n. 1, p. 199-214, 2008.

SANTOS, Boaventura Sousa. Descolonizar el saber, reinventar el poder. Montevideo: Trilce, 2009.

SARANGAPANI, Padma. Savoir, curricula et méthodes pédagogiques: le cas de l'Inde. Revue Internationale d’Éducation de Sèvres, Sèvres, 2014. Colloque L'éducation en Asie en 2014: Quels enjeux mondiaux ? Disponible en: <http://ries.revues. org/ 3848>. Acceso en: 26 oct. 2015.

SCHKOLNIK, Saúl. La princesa murta. Asución: Don Bosco, 2012.

SEPÚLVEDA, Segundo; MELLADO, María. Informe de autoevaluación pedagogía en educación general básica con mención. Temuco: Universidad Católica de Temuco, 2014.

SERRANO, Sol; PONCE DE LEÓN, Macarena; RENGIFO, Francisca. Historia de la educación en Chile (1810-1820). Barcelona: Tauro, 2012.

TUBINO, Fidel. El nivel epistémico de los conflictos interculturales. Revista Construyendo Nuestra Interculturalidad, v. 6, n. 7, p. 1-14, 2011.

TUBINO, Fidel. La praxis de la interculturalidad en los Estados Nacionales Latinoamericanos. Cuadernos Interculturales, v. 5, n. 3, p. 83-96, 2005a.

TURRA, Omar., FERRADA, Donatila; VILLENA, Alicia. La especificidad del contexto indígena como requerimiento para la formación inicial del profesorado. Revista Estudios Pedagógicos, Valdivia, $n$. 2, p. 329-339, 2013. 
TURRA, Omar; VALDEBENITO, Vanessa; TORRES, Angélica. Teaching competencies for performing in school vulnerability contexts in Chile. Journal Procedia, n. 197, p. 1236-1241, 2015.

WALSH, Catherine. Interculturalidad, estado, sociedad: luchas (de) coloniales de nuestra época. Quito: Abya Yala, 2009.

WALSH, Catherine. Significados y políticas conflictivas. Revista Nueva Sociedad, n. 165, p. 121-133, 2000.

WANSINK, Bjorn et al. Epistemological tensions in prospective Dutch history teachers' beliefs about the objectives of secondary education. The Journal of Social Studies Research, Florida, n. 1, p.1-14, 2015.

Recibido en:28.05.2016

Aprobado en: 07.12.2016

Katerin Arias-Ortega es estudiante de doctorado en Educación, Universidad Católica de Temuco (UCT). Magíster en Educación, investigadora joven del Centro de Investigación en Educación en Contexto Indígena e Intercultural (CIECII).

Segundo Quintriqueo es doctor en Educación, profesor del doctorado en Educación, de la Universidad Católica de Temuco (UCT). Profesor asociado de la UCT, investigador titular del Centro de Investigación en Educación en Contexto Indígena e Intercultural CIECll e investigador asociado del Centre Interuniversitaire d'Études et de Recherches Autochtones (ClÉRA) de I'Université Laval, Québec, Canadá.

Vanessa Valdebenito es doctora en Psicología de la educación, profesora del doctorado en Educación, Universidad Católica de Temuco. Investigadora del Proyecto №11130216, financiado por el Fondo de Desarrollo Científico y Tecnológico (FONDECYT), miembro del Grupo de Investigación sobre Aprendizaje entre Iguales, de la Universidad Autónoma de Barcelona. 